Proceedings

\title{
Halogenated 2,1,3-benzoxadiazoles as Potential Fluorescent Warheads for Covalent Protease Inhibitors ${ }^{+}$
}

\author{
Anastasiya Kuznetsova ${ }^{1}$, Philipp Klein ${ }^{2}$ and Till Opatz 2,* \\ 1 National Research Tomsk Polytechnic University, 30 Lenin Ave., Tomsk 634050, Russia; \\ akuznetsova@tpu.ru \\ 2 Johannes Gutenberg-University Mainz Institute of Organic Chemistry, Duesbergweg 10-14, Mainz D- \\ 55128, Germany; klein@uni-mainz.de \\ * Correspondence: opatz@uni-mainz.de \\ + Presented at the 22nd International Electronic Conference on Synthetic Organic Chemistry, 15 November- \\ 15 December 2018; Available Online: https://sciforum.net/conference/ecsoc-22.
}

Published: 14 November 2018

\begin{abstract}
Recently there has been a growing interest in covalent protease inhibitors in both industry and academia, caused by their longer residence times, their higher potency and their high ligand efficiency. Covalently reactive moieties which interact with activated amino acid residues such as serine or cysteine in enzymes like proteases or esterases mostly act through nucleophilic addition, substitution or ring opening. In contrast, nucleophilic aromatic substitution $\left(\mathrm{S}_{\mathrm{N}} \mathrm{Ar}\right)$ is rarely employed. In our previous work, we prepared and investigated electrophilic "warheads", which contain aromatic, heteroaromatic or quinoid fragments. Some of them show potent inhibition constants for cathepsin L, cathepsin B, rhodesain or dengue-protease, and depending on the exact nature of the electrophile they exhibit reversible covalent or irreversible inhibition modes. In the present work, we demonstrate the synthesis of fluorescent "warhead" candidates based on 2,1,3benzoxadiazoles and the investigation of their physicochemical and photophysical properties. These molecules shall serve as probes for the detailed analysis of association/dissociation mechanism and of the kinetic parameters of the bond forming event.
\end{abstract}

Keywords: 2,1,3-benzoxadiazoles; covalent protease inhibitors; fluorescentic properties

\section{Introduction}

Alkyl halides, Michael acceptors, alkyl boronates, nitriles, sulfonyl fluorides, epoxides or aziridines have been used as reactive groups for the design of covalent inhibitors of proteases or esterases [1,2]. In contrast, only a small number of compounds are known to react with proteins in an $\mathrm{S}_{\mathrm{N}} \mathrm{Ar}$-reaction. Nevertheless, the general feasibility of $\mathrm{S}_{\mathrm{N}} \mathrm{Ar}$-based covalent protein ligands has recently been demonstrated [3]. For this reason, we have synthesized a series of different aromatic, heteroaromatic or quinoid electrophilic compounds, which exhibited a strong inhibitory activity on some proteases [4].

While the latter electrophilic moieties did not show a significant fluorescence, we became interested in the design and synthesis of fluorescent $S_{N} A r$ reactive groups to investigate the association and the bond-forming event with the target enzyme in detail. It is known that derivatives of 2,1,3-benzoxadiazoles can be used as fluorescent labels for various biomolecules or -objects. For example, in our previous work, we showed the possibility of introducing fragments of 2,1,3benzoxadiazoles into nucleosides and oligonucleotides [5,6]. They are also used for the synthesis of fluorescent amino acids, glucosides or protein binders [7-9]. 
Here, we report the synthesis of new fluorescent protease inhibitor candidates based on dihalogenated 2,1,3-benzoxadiazoles and the investigation of their photophysical properties.

\section{Results and Discussion}

The synthesis of electrophilic protease inhibitors was carried out according to Scheme 1.

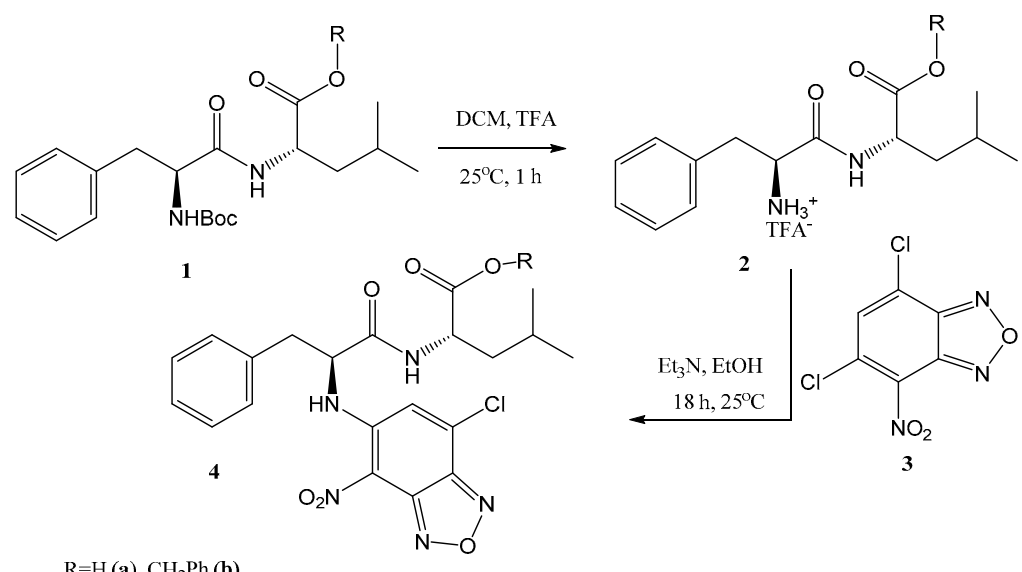

Scheme 1. Synthesis of compounds 4 .

Compounds 1 and 2 were synthesized according to a procedure by Lawesson et al. [10], compound 3 was obtained by a method developed by Boulton and Katritzky [11]. The structures of Compounds $\mathbf{4} \mathbf{a}, \mathbf{b}$ were also confirmed by physicochemical analysis methods. To assess the possibility of using substances 4 as fluorescent labels which shall allow the detailed analysis of the various stages of covalent protein inhibition, we studied the luminescent properties of these compounds. Fluorescence spectra were recorded in $\mathrm{MeOH}$ and $\mathrm{MeCN}$, shown in Figures 1 and 2. Maxima of emission are in the region of $475-485 \mathrm{~nm}$. Compounds $\mathbf{4 a , b}$ have similar curve profiles in different solvents, but there is a slight shift in maximum emissions towards the long-wave region in $\mathrm{MeCN}$.

Fluorescence Spectrum of Compound 4a

$\left(7 \cdot 10^{-5}, \mathrm{MeCN}\right)$

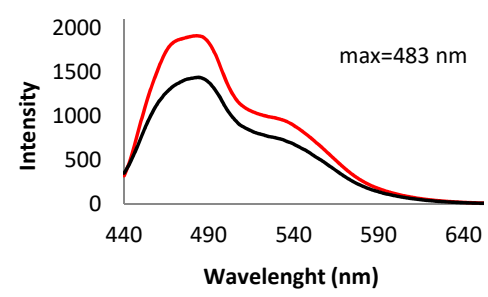

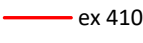

- ex 302
Fluorescence Spectrum of Compound 4a

$\left(7 \cdot 10^{-5}, \mathrm{MeOH}\right)$

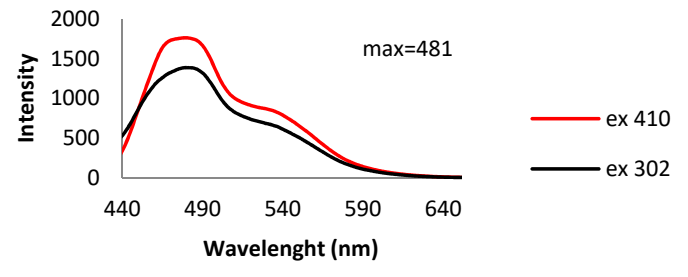

Figure 1. Fluorescence spectra of compound 4a in $\mathrm{MeCN}$ and $\mathrm{MeOH}$.
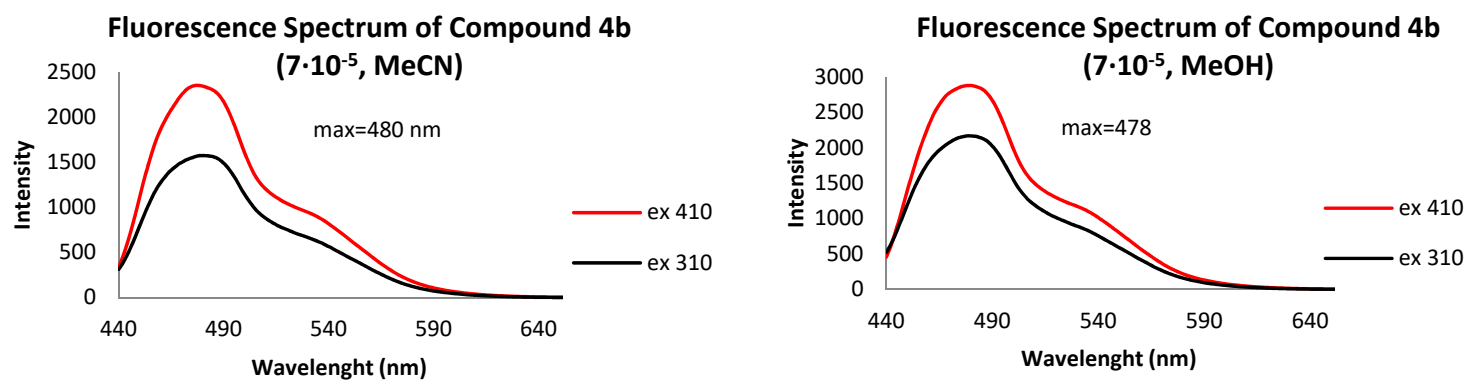

Figure 2. Fluorescence spectra of compound $\mathbf{4 b}$ in $\mathrm{MeCN}$ and $\mathrm{MeOH}$. 
The location of the maximum emissions in a relatively narrow wavelength region is possibly caused by the presence of a hydrogen bond between the N-terminal amino group of the dipeptide and the nitro group located on the benzofurazan. The existence of this bond is also confirmed by the value of the chemical shift of the $\mathrm{NH}$ proton which is found strongly downfield shifted at $10.6 \mathrm{ppm}$. To exclude the influence of the hydrogen bond on the fluorescent properties of derivatives of 2,1,3benzoxadiazoles, we synthesized a model compound from a secondary amine and studied its photophysical properties as well. 7-Chloro-5-morpholino-4-nitrobenzo[c][1,2,5]-oxadiazole (5) was synthesized according to Scheme 2.<smiles>CC(C)[C@@H]1CNCCO1</smiles>

Scheme 2. Synthesis of compound 5.

The emission maximum of compound 5 is at $546 \mathrm{~nm}$. Furthermore, we studied the $S_{N} A r$ reaction of this compound with mercaptoethanol simulating the active cysteine residues in certain proteases and also studied the impact on the fluorescent properties (Scheme 3).

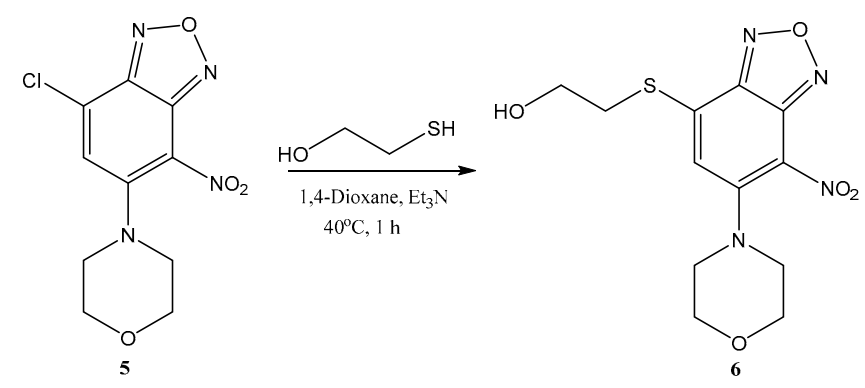

Scheme 3. Synthesis of compound 6 .

The nucleophilic displacement of the second chlorine by thiolate doesn't change the emission wavelength but increases the fluorescence intensity (the determination of the quantum yield is currently underway in our laboratory). The fluorescence spectra of compounds $\mathbf{5}$ and $\mathbf{6}$ are depicted in Figure 3.

\section{Fluorescence Spectrum of Compound 5} $\left(1 \cdot 10^{-4}, \mathrm{MeOH}\right)$

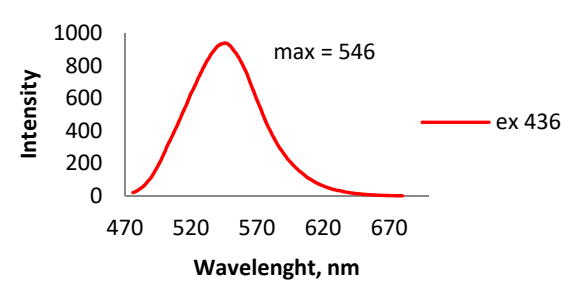

Fluorescence Spectrum of Compound 6 $\left(7 \cdot 10^{-5}, \mathrm{MeCN}\right)$

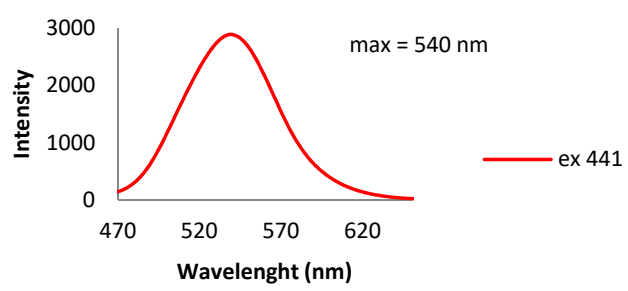

Figure 3. Fluorescence spectra of compounds 5,6.

Quantum yields were calculated relative to quinine sulfate for compounds $4 \mathrm{a}, \mathrm{b}$ and to fluorescein for compounds 5 and 6 . Values of quantum yields for all compounds are lower than $1 \%$. Such small values are possibly caused by the presence of a nitro group-adjacent to an amino function. 


\section{Methods}

All reagents and solvents were obtained from commercial suppliers (Sigma Aldrich, Alfa Aesar, TCI chemicals, ABCR, Acros Organics and Fischer Scientific) and used without further purification. Analytical thin-layer chromatography (TLC) was performed on Merck silica gel plates $\left(60 \mathrm{~F}_{254}\right)$ with defined solvent mixtures and visualized under ultraviolet (UV) light irradiation and/or TLC staining reagents. The melting points were determined in an open capillary tube. Nuclear magnetic resonance (NMR) experiments were performed on a $300 \mathrm{MHz}\left(300 \mathrm{MHz}{ }^{1} \mathrm{H}\right.$ and $75.4 \mathrm{MHz}{ }^{13} \mathrm{C}$ ) or a $600 \mathrm{MHz}$ (600 MHz ${ }^{1} \mathrm{H}$ and $153.8 \mathrm{MHz}{ }^{13} \mathrm{C}$ ) spectrometer from Bruker using deuterated solvents ((residual) solvent signals: $\left.\mathrm{CDCl}_{3}: \delta \mathrm{H}=7.26 \mathrm{ppm}, \delta \mathrm{C}=77.16 \mathrm{ppm} ;\left(\mathrm{CD}_{3}\right)_{2} \mathrm{SO}: \delta \mathrm{H}=2.50 \mathrm{ppm}, \delta \mathrm{C}=39.52 \mathrm{ppm}\right)$ as an internal reference and reported in parts per million (ppm, $\delta$ ) relative to tetramethylsilane (TMS, $\delta=0.00 \mathrm{ppm}$ ). The absorption spectra were recorded on Thermo Scientific Evolution $201 \mathrm{using}$ a $1 \mathrm{~cm}$ path length quartz cuvette. Samples were analyzed in $\mathrm{MeCN}$ or $\mathrm{MeOH}$, concentration $7 \times 10^{-5} \mathrm{~mol}$ $\mathrm{L}^{-1}$ for compounds $4 \mathbf{a}, \mathbf{b}$; in MeCN, concentration $1 \times 10^{-4} \mathrm{~mol} \mathrm{~L}^{-1}$ for compound 5 , and in $\mathrm{MeCN}$, concentration $7 \times 10^{-5} \mathrm{~mol} \mathrm{~L}^{-1}$ for compound 6 . All fluorescence spectra were recorded on a JASCO FP-8300 spectrofluorometer equipped with a xenon arc lamp using a $1 \mathrm{~cm}$ path length quartz cuvette. We used excitation (Ex) and emission (Em) bandwidths of $10 \mathrm{~nm}$ for compounds $\mathbf{4 a}, \mathbf{b}$ and Ex, Em bandwidths of $20 \mathrm{~nm}$ for compounds 5 and 6 .

(7-Chloro-4-nitrobenzo[c][1,2,5]oxadiazol-5-yl)-L-phenylalanyl-L-leucine: Crude L-Phenylalanyl-L-leucine trifluoroacetic acid salt $(51.43 \mathrm{mg}, 0.185 \mathrm{mmol}, 1$ eq.), 5,7-dichloro-4-nitrobenzo[c][1,2,5]oxadiazole ( $65 \mathrm{mg}, 0.28 \mathrm{mmol}, 1.5 \mathrm{eq}$.) were dissolved in ethanol $(5 \mathrm{~mL})$ and triethylamine $(50 \mu \mathrm{L})$ was added. After stirring for $18 \mathrm{~h}$ at room temperature, the solvent was removed under reduced pressure. Column chromatography ( $\left.\mathrm{SiO}_{2}, \mathrm{DCM}: \mathrm{MeOH} ; 20: 1\right)$ gave the pure product $(29.1 \mathrm{mg}, 61 \mu \mathrm{mol}, 33 \%)$.

${ }^{1} H \mathrm{NMR}, \operatorname{COSY}\left(600 \mathrm{MHz}, \mathrm{DMSO} d_{6}\right): \delta / \mathrm{ppm}=10.83\left(\mathrm{~s}, 1 \mathrm{H}, \mathrm{N} H^{\text {Phe }}\right), 8.77\left(\mathrm{~s}, 1 \mathrm{H}, \mathrm{N} H^{\mathrm{Leu}}\right), 7.23-7.17(\mathrm{~m}$, $\left.5 \mathrm{H}, H^{\mathrm{Ar}}, 1 \mathrm{H}, \mathrm{CH}^{5}\right), 5.19\left(\mathrm{~m}, 1 \mathrm{H}, \alpha-\mathrm{CH}^{\text {Phe }}\right), 4.36\left(\mathrm{~m}, 1 \mathrm{H}, \alpha-\mathrm{CH}^{\mathrm{Leu}}\right), 3.38\left(\mathrm{dd}, 13.7,8.8 \mathrm{~Hz}, 1 \mathrm{H}, \beta-\mathrm{CH}_{2}{ }^{\text {Phe }}\right)$, $3.06\left(\mathrm{dd}, J=13.7,8.8 \mathrm{~Hz}, 1 \mathrm{H}, \beta-\mathrm{CH}_{2}{ }^{\text {Phe }}\right), 1.64-1.23\left(\mathrm{~m}, 3 \mathrm{H}, \beta-\mathrm{CH}_{2}, \gamma-\mathrm{CH}^{\text {Leu }}\right), 0.94-0.86\left(\mathrm{~m}, 6 \mathrm{H}, \mathrm{CH}\left(\mathrm{CH}_{3}\right)_{2}\right)$.

${ }^{13} \mathrm{C} N M R, H S Q C, H M B C\left(150 \mathrm{MHz}, \mathrm{DMSOd}_{6}\right): \delta / \mathrm{ppm}=174.1(\mathrm{COOH}), 169.1(\mathrm{C}=\mathrm{O}$ Phe $), 150.1(\mathrm{C}-4 \mathrm{a})$, 145.8 (C-7a), $145.6(\mathrm{C}-7), 136.0\left(\mathrm{CH}^{\mathrm{Ar}}\right), 130.4(\mathrm{C}-6), 129.0\left(\mathrm{CH}^{\mathrm{Ar}}\right), 127.7\left(\mathrm{CH}^{\mathrm{Ar}}\right), 124.8(\mathrm{C}-5) 112.6(\mathrm{C}-4)$, $58.46\left(\alpha-\mathrm{CH}^{\mathrm{Phe}}\right), 51.0\left(\alpha-\mathrm{CH}^{\mathrm{Leu}}\right), 24.7\left(\gamma-\mathrm{CH}^{\mathrm{Leu}}\right), 23.41,21.50\left(\delta-\mathrm{CH}_{3}^{\mathrm{Leu}}\right)$.

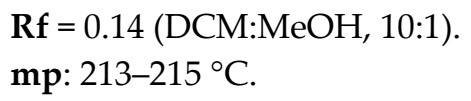

Benzyl (7-chloro-4-nitrobenzo[c][1,2,5]oxadiazol-5-yl)-L-phenylalanyl-L-leucinate: Crude benzyl Lphenylalanyl-L-leucinate trifluoroacetic acid salt (103 mg, $0.21 \mathrm{mmol}, 1$ eq.), 5,7-dichloro-4nitrobenzo[c][1,2,5]oxadiazole $(75 \mathrm{mg}, 0.32 \mathrm{mmol}, 1.5 \mathrm{eq}$.) were dissolved in ethanol $(6 \mathrm{~mL})$ and triethylamine $(100 \mu \mathrm{L})$ was added. After stirring for $18 \mathrm{~h}$ at room temperature, the solvent was removed under reduced pressure. Column chromatography $\left(\mathrm{SiO}_{2}\right.$, Cyclohexane: Ethyl acetate, 2:1) gave the pure product (109 $\mathrm{mg}, 0.20 \mathrm{mmol}, 90 \%)$.

${ }^{1} \mathrm{H} \mathrm{NMR}, \operatorname{COSY}\left(300 \mathrm{MHz}, C D C l_{3}\right): \delta / \mathrm{ppm}=10.57\left(\mathrm{~d}, J=6.7 \mathrm{~Hz}, 1 \mathrm{H}, \mathrm{N} H^{\mathrm{Phe}}\right), 7.38-7.30\left(\mathrm{~m}, 10 \mathrm{H}, H^{\mathrm{Ar}}\right)$, $6.98\left(\mathrm{~s}, 1 \mathrm{H}, \mathrm{CH}^{5}\right), 6.23\left(\mathrm{~d}, J=8.4 \mathrm{~Hz}, 1 \mathrm{H}, \mathrm{N} H^{\mathrm{Leu}}\right), 5.15\left(\mathrm{~m}, 2 \mathrm{H}, \mathrm{OCH}_{2}^{\mathrm{Bz}}\right), 4.75\left(\mathrm{~m}, 1 \mathrm{H}, \alpha-\mathrm{CH}^{\text {Leu }}\right), 4.54(\mathrm{~m}$, $\left.1 \mathrm{H}, \alpha-\mathrm{CH}^{\text {Phe }}\right), 3.39\left(\mathrm{dd}, J=14.1,4.7 \mathrm{~Hz}, 1 \mathrm{H}, \beta-\mathrm{CH}_{2}{ }^{\text {Phe }}\right), 3.29\left(\mathrm{dd}, J=14.1,4.7 \mathrm{~Hz}, 1 \mathrm{H}, \beta-\mathrm{CH}_{2}{ }^{\text {Phe }}\right), 1.70-1.50$ $\left(\mathrm{m}, 3 \mathrm{H}, \beta-\mathrm{CH}_{2}, \gamma-\mathrm{CH}^{\mathrm{Leu}}\right), 0.93\left(\mathrm{~m}, 6 \mathrm{H}, \mathrm{CH}\left(\mathrm{CH}_{3}\right)_{2}\right)$.

${ }^{13} \mathrm{C} N M R, H S Q C, H M B C$ (75 MHz, $\left.C D C l 3\right): \delta / p p m=172.0$ ( $\left.C=\mathrm{O}^{\text {Leu }}\right), 168.7$ ( $\left.C=\mathrm{O}^{\text {Phe }}\right), 149.21$ (C-4a), 145.4 (C-7a), 144.8 (C-6), 135.0 (OCC $\left.{ }^{\mathrm{Leu}}\right), 132.2(C-7), 129.4,128.6\left(\mathrm{CH}^{\mathrm{Ar}}\right), 121.9(\mathrm{C}-5), 113.3(\mathrm{C}-4) 67.5\left(\mathrm{CH}_{2} \mathrm{Bz}^{\mathrm{Bz}}\right)$, $60.5\left(\alpha-\mathrm{CH}^{\mathrm{Phe}}\right), 51.3\left(\alpha-\mathrm{CH}^{\mathrm{Leu}}\right), 41.0\left(\beta-\mathrm{CH}_{2}{ }^{\mathrm{Leu}}\right), 39.5\left(\beta-\mathrm{CH}_{2}{ }^{\mathrm{Ph}}\right), 24.9\left(\gamma-\mathrm{CH}^{\mathrm{Leu}}\right), 27.3,21.63\left(\delta-\mathrm{CH}_{3}{ }^{\mathrm{Leu}}\right)$. 
$\mathbf{R f}=0.35$ (EtOAc: Cyclohexane, 1:2).

mp: $169-170{ }^{\circ} \mathrm{C}$.

ESI-MS: $m / z=566.2\left(62 \%,[\mathrm{M}+\mathrm{H}]^{+}\right), 588.2\left(26 \%,[\mathrm{M}+\mathrm{Na}]^{+}\right)$.

UV-spectrum (MeCN, $\left.1 \times 10^{-5} \mathrm{~mol} \mathrm{~L}^{-1}\right), \lambda_{\max }, \mathrm{nm}: 302 \mathrm{~nm}, 411 \mathrm{~nm}$.

Fluorescence spectrum, $\lambda_{\max }$, nm: $478(\mathrm{MeOH}), 480(\mathrm{MeCN})$.

7-Chloro-5-morpholino-4-nitrobenzo[c][1,2,5]oxadiazole:5,7-Dichloro-4-nitrobenzo[c][1,2,5]oxadiazole (100 mg, $0.43 \mathrm{mmol}, 1$ eq.) was dissolved in ethanol ( $5 \mathrm{~mL}$ ) and morpholine ( $56 \mu \mathrm{L}, 0.65 \mathrm{mmol}, 1.5$ eq.) was added. After stirring for $2 \mathrm{~h}$ at room temperature, the solvent was removed under reduced pressure. Crystallization from ethanol afforded the title compound ( $85 \mathrm{mg}, 0.30 \mathrm{mmol}, 70 \%$ ).

${ }^{1} \mathrm{H} \mathrm{NMR}, \operatorname{COSY}\left(300 \mathrm{MHz}, \mathrm{DMSOd}_{6}\right): \delta / \mathrm{ppm}=8.20(\mathrm{~s}, 1 \mathrm{H}, \mathrm{CH}-5), 3.79\left(\mathrm{~m}, 4 \mathrm{H}, \mathrm{CH}_{2} \mathrm{O}\right), 3.58(\mathrm{~m}, 4 \mathrm{H}$, $\left.\mathrm{CH}_{2} \mathrm{~N}\right)$.

${ }^{13} \mathrm{C} N M R, H S Q C, H M B C(75 \mathrm{MHz}, C D C l 3):$ 8/ppm = 151.2 (C-4a), $146.6(C-6), 146.3$ (C-7a), $129.3(C-5)$, $126.7(C-7), 114.6(C-4), 66.63\left(\mathrm{CH}_{2} \mathrm{O}\right), 52.8\left(\mathrm{CH}_{2} \mathrm{~N}\right)$.

$\mathbf{R f}=0.48$ (DCM:MeOH, 10:1).

mp: $184-186^{\circ} \mathrm{C}$.

UV-spectrum (MeCN, $\left.1 \times 10^{-5} \mathrm{~mol} \mathrm{~L}^{-1}\right), \lambda_{\max }, \mathrm{nm}: 312 \mathrm{~nm}, 440 \mathrm{~nm}$.

Fluorescence spectrum, $\lambda_{\max }, \mathrm{nm}: 546(\mathrm{MeOH})$.

2-((6-Morpholino-7-nitrobenzo[c][1,2,5]oxadiazol-4-yl)thio)ethan-1-ol:

7-Chloro-5-morpholino-4-nitrobenzo[c][1,2,5]oxadiazole (22 mg, $78 \mu \mathrm{mol}, 1$ eq.), mercaptoethanol $(8.3 \mu \mathrm{L}, 0.12 \mathrm{mmol}, 1.5$ eq.) were dissolved in 1,4-dioxane $(3 \mathrm{~mL})$ and 1 drop of trimethylamine was added. After stirring for $1 \mathrm{~h}$ at $40^{\circ} \mathrm{C}$, the solvent was removed by co-evaporation with cyclohexane (5 mL). Crystallization from propanol-2 afforded the title compound ( $20 \mathrm{mg}, 61 \mu \mathrm{mol}, 79 \%$ ).

${ }^{1} \mathrm{H} N M R, \operatorname{COSY}\left(300 \mathrm{MHz}, \mathrm{DMSO} \mathrm{d}_{6}\right): \mathrm{\delta} / \mathrm{ppm}=7.34(\mathrm{~s}, 1 \mathrm{H}, \mathrm{CH}-5), 5.24(\mathrm{t}, J=5.6 \mathrm{~Hz}, 1 \mathrm{H}, \mathrm{OH}), 3.80(\mathrm{~m}$, $\left.6 \mathrm{H}, \mathrm{CH}_{2} \mathrm{O}, \mathrm{CH}_{2} \mathrm{OH}\right), 3.60\left(\mathrm{~m}, 4 \mathrm{H}, \mathrm{CH}_{2} \mathrm{~N}\right), 3.46\left(\mathrm{~m}, 2 \mathrm{H}, \mathrm{CH}_{2} \mathrm{~S}\right)$.

${ }^{13} \mathrm{C} \mathrm{NMR}, \mathrm{HSQC}, \mathrm{HMBC}\left(75 \mathrm{MHz}, \mathrm{CDCl}_{3}\right): \delta / \mathrm{ppm}=151.7(\mathrm{C}-7 \mathrm{a}), 146.3(\mathrm{C}-6), 146.2(\mathrm{C}-4 \mathrm{a}), 136.3(\mathrm{C}-4)$, $120.5(C-5), 114.7(C-7), 66.44\left(\mathrm{CH}_{2} \mathrm{O}\right), 59.68\left(\mathrm{CH}_{2} \mathrm{OH}\right), 52.87\left(\mathrm{CH}_{2} \mathrm{~N}\right), 34.36\left(\mathrm{CH}_{2} \mathrm{~S}\right)$.

$\mathbf{R f}=0.29$ (Toluene:EtOAc, 4:1).

mp: $177-179{ }^{\circ} \mathrm{C}$.

UV spectrum (MeCN, $\left.1 \times 10^{-5} \mathrm{~mol} \mathrm{~L}^{-1}\right), \lambda_{\max }, \mathrm{nm}: 372 \mathrm{~nm}, 441 \mathrm{~nm}$.

Fluorescence spectrum, $\lambda_{\max }, \mathrm{nm}: 540(\mathrm{MeCN})$.

\section{Conclusions}

Compounds $4 \mathbf{a}, \mathbf{b}$ were synthesized as potential protease inhibitors allowing a multiparameter optical read-out, and their photophysical properties were investigated. The emission maxima of these compounds are located at relatively short wavelengths due to the presence of an intramolecular hydrogen bond. In contrast, derivatives devoid of NH-protons have longer emission wavelengths and a second $S_{N} A r$ reaction with a thiolate leads to an increase in the fluorescence intensity while keeping the emission wavelengths essentially unchanged.

Acknowledgments: The reported study was funded by Ministry of education and science of Russian Federation according to the research project № 412754.2018/12.2 and DAAD according to the research № 91687868. We thank the Rhineland-Palatinate Center for Natural Products Research and Tanja Schirmeister (Mainz) for the helpful discussion as well a for biological evaluation of the initial compound series (data not shown). 


\section{References}

1. Schirmeister, T. Inhibition of cysteine proteases by peptides containing aziridine-2, 3-dicarboxylic acid building blocks. Biopolymers 1999, 51, 87-97.

2. Powers, J.C.; Asgian, J.L.; Ekici, Ö.D.; James, K.E. Irreversible inhibitors of serine, cysteine, and threonine proteases. Chem. Rev. 2002, 102, 4639-4750.

3. Shanon, D.A.; Banerjee, R.; Webster, E.R.; Bak, D.W.; Wang, C.; Weerapana, E. Investigating the Proteome Reactivity and Selectivity of Aryl Halides.J. Am. Chem. Soc. 2014, 136, 3330-3333.

4. Klein, P.; Johe, P.; Jung, S.; Kesselring, J.; Tenzer, S.; Distler, U.; Anh Le, T.; Engels, B.; Hellmich, U.A.; Opatz, T.; et al. Aromatic Warheads for Cysteine Protease Inhibitors, in preparation.

5. Kupryushkin, M.S.; Konevet, D.A.; Vasilyeva, S.V.; Kuznetsova, A.S.; Stetsenko, D.A.; Pyshnyi, D.V. Oligonucleotide Functionalization by a Novel Alkyne-Modified Nonnucleosidic Reagent Obtained by Versatile Building Block Chemistry. Nucleos. Nucleot. Nucl. Acids 2013, 32, 306-319.

6. Vasilyeva, S.V. Novel Fluorescent Pyrimidine Nucleosides Containing 2, 1, 3-Benzoxadiazole and Naphtho-[1, 2, 3-CD] Indole-6 (2H)-One Fragments. Nucleos. Nucleot. Nucl. Acids 2014, 33, 615-625.

7. Zhao, Y.; Pirrung, M.C.; Liao, J. A fluorescent amino acid probe to monitor efficiency of peptide conjugation to glass surfaces for high density microarrays. Mol. BioSyst. 2012, 8, 879-887.

8. Wenskowsky, L.; Schreuder, H.; Derdau, V.; Matter, H.; Volkmar, J.; Nazaré, M.; Opatz, T.; Petry, S. Identification and Characterization of a Single High-Affinity Fatty Acid Binding Site in Human Serum Albumin. Angew. Chem. Int. Ed. 2018, 57, 1044-1048.

9. Yi, H.; Maisonneuve, S.; Xie, J. Synthesis of triazole-linked fluorescent saccharides and glycosyl amino esters. Synthesis 2012, 44, 1647-1656.

10. Clausen, K.; Thorsen, M.; Lawesson, S.O.; Spatola, A.F. Synthesis of (R)-and (S)-(glu) thz and the corresponding bisthiazole dipeptide of dolastatin 3. J. Chem. Soc. Perkin Trans. 1984, 1, 785.

11. Boulton, A.J.; Gray, A.C.G.; Katritzky, A.R. Heterocyclic rearrangements. Part VIII. Attempted intramolecular oxygen-transfer in chlorofurazanobenzofuroxans. J. Chem. Soc. B 1967, 9, 909-911. 